\title{
Algunos enlaces conceptuales entre psicopatología del desarrollo, personalidad y evaluación psicológica infantojuvenil
}

\author{
Some conceptual links between psychopathology of development, personality and psychological \\ assessment of children and adolescents
}

\section{E. Norma Contini ${ }^{1}$}

${ }^{1}$ Facultad de Psicología, Universidad Nacional de Tucumán. Argentina

\begin{abstract}
Resumen: Objetivos: 1) analizar conceptos sobre psicopatología infantojuvenil que permitan abordar la normalidad/anormalidad del comportamiento. Se deslindan conceptos de temperamento, carácter y personalidad. 2) considerar donde situar los trastornos que se detectan en el área emocional en la infancia. Se discute si corresponde hacerlo bajo el concepto de personalidad. Se desarrollan teorías de las que han derivado pruebas de evaluación de la personalidad en niños y adolescentes. Se concluye que no hay datos empíricos suficientes que permitan aseverar la estabilidad del temperamento; sería prudente conceptualizar la personalidad como estructura a partir de la adolescencia; en la infancia sería factible enunciarla solo en torno a dimensiones. La comunicación supone una reflexión crítica de conceptos de psicopatología del desarrollo, trastornos emocionales de inicio en la infancia y su vinculación con el concepto de personalidad que se presentan dispersos en la literatura y que sirven de respaldo a la evaluación psicológica.
\end{abstract}

Palabras clave: psicopatología, niñez, adolescencia, personalidad; problemas emocionales, evaluación psicológica

\begin{abstract}
Objectives: 1) To provide concepts on child-adolescent psychopathology that allow an approach to the behavioral normality/abnormality. Concepts of temperament, character and personality are defined. 2) To analyse where to locate the disorders that are detected in the emotional area in childhood. It is discussed whether to do so under the concept of personality. To develop theories that have derived personality assessment tests in children and adolescents. It is concluded that there is insufficient empirical data to assert the temperament's stability; it would be prudent to conceptualize personality as a structure of adolescence. In childhood it would be feasible to enunciate it only around dimensions. The work offers a critical analysis of concepts of developmental psychopathology, emotional disorders of initial childhood and their relationship with the concept of personality that is scattered in the literature and supports psychological assessment.
\end{abstract}

Key words: psychopathology; childhood; adolescent; personality; emotional problems, psychological assessment

Cómo citar esta comunicación:

Contini, E. N. (2018). Algunos enlaces conceptuales entre psicopatología del desarrollo, personalidad y evaluación psicológica infantojuvenil. Ciencias Psicológicas,12(1), 147-157.

doi: https://doi.org/10.22235/cp.v12i1.1604

Correspondencia: E. Norma Contini. Facultad de Psicología, Universidad Nacional de Tucumán, Argentina. San Juan 150, 3er.p., Dpto.5. (4000).S.M. de Tucumán, e-mail: contini.norma@gmail.com 


\section{Algunos enlaces conceptuales entre psicopato- logía del desarrollo, personalidad y evaluación psicológica infantojuvenil}

Las tareas de evaluación psicológica en el campo de la infancia y adolescencia se han consolidado en las últimas décadas. Este desarrollo está en íntima conexión con la comprobación que la intervención temprana - apoyada en la información que provee la evaluación psicológica - evita la instalación y cristalización posterior de cuadros psicopatológicos. El uso de pruebas construidas y validadas con rigor metodológico ha sido fundamental. Igualmente decisivos han sido los aportes de teorías como la sistémica, cognitiva y los desarrollos de las neurociencias. La complejidad de asumir esta tarea reside en que el niño / adolescente es un sujeto en desarrollo. Se hace imprescindible deslindar los comportamientos esperables de los no esperables. En tal sentido los aportes de la psicopatología infantojuvenil constituyen un pilar básico desde donde respaldar hipótesis diagnósticas.

El objetivo de este trabajo es, a partir de una síntesis de conceptos sobre psicopatología infantojuvenil que sean útiles, pensar la anormalidad /normalidad del comportamiento del niño y del adolescente, o dicho de otro modo, el grado de ajuste del comportamiento según lo que se espera de acuerdo a la edad cronológica y contexto ecológico y cultural. De lo contrario se corre el riesgo de operar en un vacío conceptual, que no podrá ser remediado con un abordaje metodológico, por riguroso que sea. Un segundo objetivo es analizar si es posible conceptualizar en la infancia y adolescencia la personalidad, estableciendo conexiones entre ésta y los incipientes trastornos psicopatológicos. Los conceptos que se discuten en este trabajo forman parte del marco teórico del Proyecto de investigación Adolescentes en riesgo: indicadores de agresividad y vulnerabilidad psicopatológica. ${ }^{l}$

Tal como señala Jiménez Hernández (1995), Campbell (1990), Rodríguez Sacristán, Lozano Oyola \& Rodríguez-Sacristán Cascajo (2005), González Barrón \& Montoya Castilla (2015) no abundan los textos sobre psicopatología infantil y sus desarrollos han sido siempre más tardíos que los referidos a adultos. En tal sentido puede

1 Proyecto de Investigación, (Plan de Mejoras en Psicología, PMP). Facultad de Psicología, Universidad Nacional de Tucumán, Argentina. Subsidio: Sec. Políticas Universitarias (2016-2018). afirmarse que la Psicopatología Infantil es propia del siglo XX y que las relaciones con la Psicología Clínica y la Psicología del Desarrollo son recientes. Tales teorizaciones han ocurrido a partir de la aceptación de que la infancia es un período del ciclo vital distinto del adulto y, más recientemente, por el reconocimiento del niño como un sujeto de derechos. Un tema crítico, aún en estudio rudimentario es el papel que juega el desarrollo y de qué modo puede derivar en problemas psicopatológicos (Schoeps y González Barrón, 2015). Existe coincidencia en que las líneas actuales de investigación se centran en los estudios longitudinales, que aportan datos muy importantes sobre la continuidad o discontinuidad en psicopatología entre la infancia y la edad adulta. Igualmente, los estudios epidemiológicos (Jiménez Hernández 1995; Schoeps \& González Barrón, 2015) hacen posible formular hipótesis causales acerca de cómo evoluciona la psicopatología a lo largo del ciclo vital. Así también han cobrado relevancia los estudios sobre la incidencia de factores sociales, de tal modo que la interrogante gira en torno al papel de la familia, la escuela, el grupo de pares y la comunidad misma en la generación de salud o psicopatología en esta etapa.

\section{Cómo conceptualizar la psicopatología infantojuvenil}

Siguiendo a Luciano (1997) y Jiménez Hernández (1995) puede conceptualizarse a la Psicopatología infantojuvenil como aquella disciplina que estudia el comportamiento anormal, o alteraciones en el comportamiento en el niño / adolescente. Se propone explicar dicho comportamiento y los factores responsables de tales alteraciones.

Almonte (2012) la define como aquella disciplina cuyo objetivo es el estudio de las organizaciones y dinámicas psicológicas que subyacen a las perturbaciones del psiquismo, con el propósito de conocer sus determinantes, evolución y consecuencias que un trastorno puede ocasionar en el desarrollo del niño/adolescente.

La particularidad de la psicopatología infantojuvenil es que realiza estudios tanto a nivel transversal (enfoque sincrónico) de un fenómeno psíquico, como longitudinal (enfoque diacrónico) con el propósito de constatar posibles cambios que ocurren de acuerdo al desarrollo. 
La definición de lo anormal adquiere complejidad porque se debe tener en cuenta la variable del desarrollo. Si bien el desarrollo en la infancia y adolescencia presenta diversas secuencias universales, no es menos cierto que dicho desarrollo adquiere particularidades según el contexto cultural. En tal sentido la Psicología Transcultural ha hecho aportes sustanciales a la comprensión de la diversidad/universalidad del comportamiento (Contini, 2013; Georgas, van de Vijver \& Berry, 2004). Como afirma Berry, Poortinga, Breugelmans, Chaisotis \& Sam (2011) una cuestión central en inacabado debate en Psicología Transcultural refiere hasta qué punto las funciones psicológicas y los procesos subyacentes son comunes a la condición humana (Universalismo) o, por el contrario, son específicos de ciertos grupos culturales (Relativismo).

Siguiendo a Jiménez Hernández (1995) se tendrán en cuenta los siguientes factores para identificar el comportamiento normal o patológico:

a) Valoración del comportamiento en el contexto del desarrollo: una característica central en el niño es la movilidad de los estilos de comportamiento; por ende, un comportamiento puede ser normal en una etapa y no serlo en otra. La edad cronológica (EC) es básica para definir el problema, por ejemplo, las dificultades en el habla a los 3 años de EC no tienen la misma significación que a los 10 años de EC. Por lo tanto, las normas propias del desarrollo son un criterio ineludible para evaluar los comportamientos. En el mismo sentido Ross (1980) señala que tener en cuenta el desarrollo supone la observación del comportamiento de grupos de niños de la misma edad y representativos de una población específica. A su vez, Rodríguez Sacristán et al (2005) alude a cronodependencia, es decir que las organizaciones psicopatológicas se estructuran según las edades. Son diversos los criterios que se emplean para dilucidar si un comportamiento se encuentra dentro de lo esperado. Uno de ellos es el estadístico (Jiménez Hernández, 2005), otro criterio es el cultural en un sentido amplio, lo que implica tener en cuenta diversos contextos y subculturas.

Tomando a Ross (1980) se dirá que se considera perturbado al comportamiento del niño que se aleja de normas sociales propias de un grupo y que ocurre con una frecuencia e intensidad que los adultos que rodean al niño consideran como excesiva o insuficiente. De esta definición se destaca la necesidad de tener en cuenta tres aspectos primordiales: frecuencia, duración e intensidad del comportamiento (Jiménez Hernández, 1995), lo cual lleva a considerar si se dan por exceso o por defecto.

b) Nivel de desarrollo cognitivo: el nivel de desarrollo cognitivo alcanzado por el niño influye en la valoración que los adultos hacen del comportamiento de aquel. Por ejemplo, no se atribuirá el mismo significado si un niño de 3 años no acepta compartir un juguete con otro niño, pudiendo inclusive agredirlo si este lo toma para jugar, que si el niño tiene 8 años y se vincula con sus pares solo por medio de la agresión.

c) La valoración del comportamiento es realizada por adultos: son principalmente los padres, maestros o cuidadores que conviven con el niño quienes valoran el comportamiento en base a normas propias del grupo social en el cual están inmersos Este es un tema crítico, pues no será igual la valoración que puede hacer una madre perfeccionista y exigente, que otra de estilo laisse faire. Así es como en evaluación psicológica infantil se trabaja con multinformantes, y muchas veces no hay coincidencia en los juicios de valor que provienen de padres, otros familiares o maestros.

d) Criterio situacional: un comportamiento puede ser adecuado en un contexto y en una situación, y no en otro, por ejemplo, el modo de vincularse verbalmente los compañeros en el recreo y en una hora de clase.

\section{Psicopatología del desarrollo}

En función de los argumentos planteados precedentemente respecto al peso que las variables del desarrollo tienen cuando se debe definir si un comportamiento es esperable o normal, se ha desarrollado una disciplina que se conoce como Psicopatología del Desarrollo. Sroufe \& Rutter (1984) la definen como "el estudio de los orígenes y curso de los patrones individuales de la no adaptación del comportamiento sea cual fuere la edad de comienzo, sus causas, las transformaciones en la manifestación de dicho comportamiento y lo complejo del curso del patrón evolutivo" (p. 18).

Como bien ha planteado Rodríguez Sacristán et al (2005) la psicopatología del desarrollo ha tenido el mérito de interconectar criterios del desarrollo con aportes de la Psicología Clínica.

El objetivo de la psicopatología del desarrollo es estudiar el comportamiento según la secuencia del desarrollo en el orden físico, cognitivo y 
socioemocional. Es también un objetivo central estudiar cómo se produce la integración entre los sistemas biológico, psicológico y social del niño/ adolescente para poder explicar tanto el comportamiento adaptado como el desadaptado. Se parte del supuesto que la vulnerabilidad para los trastornos psicológicos se vincula a la cualidad de la organización entre todos estos sistemas y no de algún componente aislado (Lemos Giráldez, 2003). Desde este enfoque se admite la existencia de estructuras psicopatológicas cambiantes a través del tiempo. El niño/adolescente tiene capacidad de autorregulación, conforme ocurre el proceso de maduración. A partir de unas bases biológicas, e inmerso en un contexto social, se observan periodos de organización y otros de desorganización del aparato psíquico, que siempre se encuentra en situaciones de adaptación, en ocasiones con riesgos de no seguir el curso que se espera. Otra característica propia de la psicopatología del desarrollo es la comorbilidad (Rodríguez Sacristán et al, 2005), es decir que las organizaciones psicopatológicas rara vez ocurren solas, sino que se acompañan de otros síntomas o trastornos. Así, un niño de 7 años, agresivo en su modo de vinculación con los pares en el aula, puede presentar al mismo tiempo déficit de atención.

Otro aspecto que considera la psicopatología el desarrollo es la relación de continuidad- discontinuidad a largo plazo entre las organizaciones psicopatológicas del niño y el adulto (Rodríguez Sacristán et al, 2005) y se plantea si dichas manifestaciones psicopatológicas han sido superadas, mantenidas o acrecentadas. Se preocupa por estudiar si el desarrollo presenta regresiones, inhibiciones o signos de desviaciones. En esta línea, Almonte y Montt (2012) señalan, citando datos de la OMS que el 50\% de adultos con patología psiquiátrica comenzó su desarrollo en la niñez o adolescencia.

Igualmente se plantea que las manifestaciones clínicas del desarrollo psíquico anormal expresan la coexistencia de áreas de funcionamiento perturbado y áreas de funcionamiento normal que hacen posible la adaptación. Este concepto de la psicopatología infantojuvenil, sintoniza plenamente con la concepción actual de la evaluación psicológica, interesada en identificar síntomas y potencial psicopatología, pero también áreas saludables y libres de conflicto.

A su vez, cabe destacar que la psicopatología del desarrollo entiende al comportamiento normal
/ anormal como variaciones dentro de un continuo de características y no como fenómenos dicotómicos (Lemos Giraldez, 2003). En esta misma línea Achenbach (1992) alude que los comportamientos desviados, motivo de la consulta psicológica, serían variaciones cuantitativas y no cualitativas de las características que pueden ser normales en cierto periodo del desarrollo.

\section{¿Es posible conceptualizar la personalidad en este ciclo vital?}

Como bien han señalado numerosos investigadores como Eysenck (1992, 2007), Millon (1990; 1999; 2010) o Páez \& Casullo (2000) el concepto de personalidad ha sido objeto de discusión durante décadas, sin lograrse una definición con aceptación universal; más aún si se trata de niños. Millon se interroga acerca de cuáles son los límites del concepto de personalidad y cómo se puede distinguir de otros conceptos relacionados, cuyos significados se solapan y hasta se utilizan como sinónimos. A ello hay que agregar que no existe consenso entre los estudiosos de la infancia, si es posible situar los incipientes trastornos psicopatológicos en una matriz denominada personalidad; es por lo que, en este apartado se tratarán de deslindar los conceptos de temperamento, carácter y personalidad y se discutirá si es posible estudiarlos en la infancia y adolescencia.

Los constructos carácter y temperamento se han utilizado con frecuencia, al decir de Millon (1999), como términos intercambiables en la literatura sobre la personalidad. Se señala con énfasis, que el temperamento sería el modo inicial de respuesta del niño; va dotándolo de una sensibilidad que incidirá en cómo afrontará los estímulos, en cómo va a procesar la información y en el tipo de reactividad ante determinados hechos (Del Barrio Gándara, 2005; Castro Solano, 2015). Se trataría de un rasgo bastante estable, componente y base de la personalidad del adulto. Conceptualmente precisos resultan los planteos de Thomas, Chess $\&$ Birch (1968), quienes argumentan en la misma línea, señalando que el temperamento es un patrón de conducta con el que nace el niño. Tendría una base biológica y se modificaría con las interacciones del pequeño con el medio ambiente. Rothbart (2007), reconocida estudiosa del tema asume una postura biologisista, al considerar que las respuestas del niño implican actividad eléctrica cerebral y calidad de neurotransmisores; todo ello va a 
generar un particular modo de funcionamiento del sistema nervioso central y autónomo. Esta investigadora argumenta que el temperamento da cuenta de formas iniciales de configuración de la personalidad; ésta se estructurará por la conjunción del temperamento y las experiencias de vida. Ello implica el desarrollo de la cognición acerca del sí mismo, de los otros, del mundo físico y social, tanto como los valores, actitudes y estrategias de afrontamiento.

Por otra parte, Rothbart \& Bates (2006) hacen un aporte al conocimiento de la naturaleza y componentes del temperamento, señalando que se debe tener en cuenta: a) nivel de actividad, en el caso del niño se dirá la energía con que funciona, o a la inversa, el sedentarismo; b) sociabilidad, es decir qué tipo de interacciones tiene con otros y c) emocionalidad, definida como la intensidad de la respuesta afectiva.

Millon (1990, 1999, 2001), uno de los investigadores contemporáneos más destacados en el campo de la personalidad señala que la noción de temperamento -desde su aparición en lengua inglesa en la Edad Media (Millon, 1999)- se asoció al sustrato biológico del cual emergería luego la personalidad. Alude a variables constitucionales, en el sentido de determinantes bioquímicos, endocrinológicos y neurológicos que subyacen al modo de responder a los estímulos. Agrega que, el temperamento y las experiencias tempranas afectan el desarrollo y naturaleza de ciertas estructuras y funciones psicológicas. A partir de características iniciales del sujeto - actitudes, afectos, mecanismos de defensa - ocurren experiencias vitales que determinan la adquisición de nuevos atributos psicológicos relacionados causalmente con aquellas características iniciales del niño.

En función del objetivo de esta reflexión de dilucidar si es posible conceptualizar la personalidad en la infancia, merece profundizarse en los patrones iniciales de comportamiento del niño. Con relación al temperamento, Millon (1999; 2004) profundiza su planteo al decir que cada niño nace con un patrón particular de tendencias de respuestas. Afirma que dichos patrones de comportamiento, que se observan en los primeros meses de vida parecen ser de origen biogenético más que psicógenos. $\mathrm{Y}$ hay quienes hacen referencia a patrones primarios, puesto que se manifiestan antes de que el aprendizaje posnatal pueda influir en ellos. Respalda sus aseveraciones en investigaciones con niños que han contribuido a la com- prensión del desarrollo de la personalidad. Cita así diferencias en la regularidad de funciones biológicas, tales como reactividad autónoma (respuestas iniciales a nuevas situaciones); alerta sensorial a estímulos, adaptabilidad al cambio; estados de ánimo característicos e intensidad de respuestas, distractibilidad y persistencia o cualidad del tono emocional (Goldsmith \& Gottesman, 1981). El interrogante es si estas diferencias individuales iniciales provocarán una cualidad de experiencias vitales posteriores. Así, cabe preguntarse si tales características del niño pueden generar reacciones distintas en los padres. En lugar de preguntarnos qué efecto tiene el medio ambiente en el niño, el interrogante debiera plantear el efecto que el niño tiene sobre el medio y por ende, cuáles de esas consecuencias afectan su desarrollo.

T. Millon $(1990,2004)$ en concordancia con otros investigadores, tales como Kagan (1989), considera que esos patrones iniciales se modifican poco entre la infancia y la niñez y pareciera ser que las experiencias de vida reforzaran las características que se manifiestan al comienzo de la vida. Los comportamientos del bebé y del niño pequeño determinarán efectos en el entorno que acentuarían los comportamientos iniciales de aquel.

Un tema central de debate con relación al temperamento es la estabilidad de este patrón de comportamiento a lo largo del tiempo. Diversos estudios plantean la estabilidad a partir de cierta edad. Se podría situar la misma a los 2 años, según afirman Mathiesen \& Tabs, (1999) y Bates \& Bayle (1988).

Como contrapartida, otras investigaciones se centran en la modificación del temperamento por la interacción con el medio ambiente. Desde esta perspectiva serán fundamentales los estilos de crianza (afectividad, estilo comunicacional), y el propio carácter del padre y de la madre. En un sentido amplio cuenta la cultura como variable de incidencia en la propia organización familiar (Del Barrio Gándara, 2005).

El carácter por su parte es un término que se empleó inicialmente para designar lo que distinguía a una persona, en términos de una especie de marca propia. El carácter, según Millon (1990) refiere a cualidades personales vinculadas a valores y costumbres de una comunidad. El uso europeo de este constructo se asemeja más al de personalidad.

Habiendo deslindado los conceptos de temperamento y carácter, se intentará conceptualizar la personalidad. Ha variado a lo largo de la his- 
toria y según Millon hoy se concibe como "un patrón complejo de características psicológicas profundamente arraigadas, que son en su mayor parte inconscientes, y difíciles de cambiar, y se expresan automáticamente en casi todas las áreas de funcionamiento del individuo" (Millon, 1999, pág. 4). Estos rasgos parecieran emerger de una matriz compleja de factores biológicos y de aprendizajes que constituirían el patrón propio del sujeto de percibir, sentir, pensar y comportarse.

A su vez, se puede abordar la conceptualización de la personalidad desde las perspectivas nomotética e ideográfica. El enfoque nomotético busca individualizar unidades fundamentales de la personalidad, investigando regularidades que puedan ser aplicables a numerosos sujetos, y a tales regularidades se las identifica mediante el análisis factorial. De este modo, la personalidad puede ser definida como la desviación de la media del grupo con relación a determinadas organizaciones psicológicas; la combinación de varios niveles de diferencias individuales constituiría la singularidad propia de cada sujeto.

Como contrapartida, el enfoque ideográfico se centra en la individualidad. La personalidad sería una combinación única entre factores biológicos y contextuales.

Teniendo en cuenta la diversidad de teorías y enfoques existentes sobre la personalidad y, siendo nuestro foco de interés la infancia y adolescencia, se analizarán algunos conceptos centrales de teorías de las que han derivado pruebas de evaluación de la personalidad en niños y adolescentes. Así se hará referencia a teorías empíricas factoriales, como las de Eysenck y la de Costa \& McCrae; a los aportes de Achenbach y se concluirá con los desarrollos de Millon, por tratarse de una teoría integradora.

Una de las teorías factoriales, de carácter biologicista, que sitúa al temperamento como los cimientos de la personalidad, es la formulada por el Eysenck (2007). Propone tres dimensiones constitutivas de la personalidad: neuroticismo (o Inestabilidad emocional); introversión / extroversión y dureza emocional (Psicoticismo). Estas categorías encuentran sus fuentes en Jung y Kretschmer.

Para Eysenck (1992), las respuestas del sujeto a los estímulos están vinculadas a la capacidad de reacción del sistema nervioso autónomo. Este investigador construye una prueba para evaluar tales dimensiones, tanto para adultos, el Cuestionario de Evaluación de la Personalidad (EPQ-A), como para niños, el (EPQ-J). En la definición de los constructos no diferencia adultos de niños, salvo en dureza emocional, lo cual se considera una limitación. Si se parte del supuesto que el niño es un sujeto en desarrollo y que los comportamientos presentan variaciones que los diferencian de los adultos, sería necesario que estuvieran reflejados en la definición de los constructos y en la prueba específica para niños (EPQ-J).

En segundo lugar, conduce a pensar que, si construyen pruebas para niños, sería posible conceptualizar la personalidad en este ciclo vital. Ahora bien, se alude a la misma desde un enfoque empírico, es decir, las dimensiones de las que está compuesta la personalidad han sido identificadas por medio del análisis estadístico de datos derivados de la prueba. En el diseño de dichas pruebas estarían representadas partes del constructo.

Entre los componentes de la personalidad Eysenck define la dimensión emocionalidad (o neuroticismo, designada $N$ ) como propia de sujetos preocupados, con cambios de humor y emotivos (Eysenck \& Eysenck, 2007). Pueden presentar trastornos en el sueño y quejas psicosomáticas. Con relación a la dimensión extroversión /introversión este autor sigue la línea de Jung; el sujeto extrovertido es descripto como sociable, con una red variada de amigos, le agrada la excitación, el riesgo; en general es impulsivo, disfruta de las bromas y le da placer el cambio, suele ser despreocupado, optimista y está orientado a la actividad. Es probable que se ofusque con facilidad, a la vez que no logra un control de sus sentimientos. No suelen ser sujetos altamente confiables. Como contrapartida, el sujeto introvertido es retraído, reservado, tranquilo, introspectivo, distante, salvo con sus allegados íntimos. Eysenck agrega que es más proclive a disfrutar de la lectura que de las personas, piensa antes de actuar, no le atrae la diversión, tomándose con mucha seriedad los hechos de la vida cotidiana. El sujeto introvertido suele ser algo pesimista y tiene un elevado respeto por las normas. Se caracteriza por ser muy controlado en la expresión de sus sentimientos y muy confiable para su entorno.

La dimensión dureza emocional fue designada inicialmente como Psicoticismo; este último término resulta, a todas luces inadecuado en el contexto científico actual. Eysenck define como dureza emocional en el adulto, a un sujeto solitario, a quien no le preocupan los otros, pudiendo ser cruel e insensible. Se presenta hostil, agresivo 
y carente de empatía, inclusive con las personas afectivamente próximas a él. Suele tener inclinación por temas extravagantes y no puede valorar el riesgo de sus actos. Tiende a crear problemas a otros y no logra consensos con facilidad. Es la única dimensión en la cual Eysenck hace descripciones diferenciadas entre adultos y niños. Con respecto a los niños los describe como solitarios y "raros" (2007: pág. 14), carentes de sentimientos con compañeros o animales. Suelen ser agresivos, hostiles y el proceso de socialización no siempre es eficaz con ellos. El sentimiento de culpa o la sensibilidad para con los demás, son conceptos extraños para estos niños.

Eysenck ha utilizado el análisis factorial para discriminar las tres dimensiones de la personalidad que propone. En tal sentido, su teoría se inscribe dentro de las denominadas monotaxonómicas empíricas (Castro Solano, 2015). Identifica dimensiones de la personalidad, aun en niños, desde el enfoque de rasgos. No está haciendo referencia a la personalidad desde la concepción de una estructura. A nuestro criterio, definir la personalidad en términos de una estructura, de una matriz, sería propio en adultos o adolescentes, una vez que ya no tiene peso la variable del desarrollo.

En la década del ' 80 del siglo XX McCrae $\&$ Costa (1987) formulan el modelo de los cinco factores o Big Five, que se sitúa entre las teorías politaxonómicas empíricas (Castro Solano, 2005). Plantean desde la teoría de los rasgos, que los distintos modos como los sujetos se expresan desde un punto de vista emocional, de relaciones interpersonales, en sus actitudes y motivaciones son constantes y pueden sintetizase en cinco factores. Proponen un modelo que incluye las dimensiones: extroversión $(\mathrm{E})$, neuroticismo $(\mathrm{N})$, agradabilidad (A), apertura a la experiencia $(\mathrm{O}) \mathrm{y}$ escrupulosidad (consciencia y rectitud, C). Se han llevado a cabo estudios transculturales en adultos utilizando el cuestionario creado por estos autores y se concluyó que existiría una estructura universal de la personalidad (Páez y Casullo, 2000). Con relación a la estabilidad de las dimensiones de la personalidad a lo largo del tiempo, es decir entre infancia y adultez, se han realizado estudios longitudinales con resultados contradictorios. Mientras algunos encontraron estabilidad en los rasgos (Lemos, 2006), otros (Del Barrio, 2005) descubrieron variabilidad en los resultados.

Por otra parte, dentro de los estudios factoriales empíricos focalizados en la organización temprana de variables emocionales, se destacan los realizados por Achenbach (1992, 2008). No se muestra interesado en identificar rasgos de personalidad, pero hace un aporte sustancial a la comprensión de las tempranas organizaciones emocionales y estilos de orientación interpersonal, que pueden derivar luego en determinados patrones de personalidad en la adultez. Achenbach desarrolla un modelo con base empírica y clínica. Argumenta la existencia de patrones amplios de problemas infantiles, denominados externalizantes e internalizantes. Los problemas internalizantes son comportamientos con contenido emocional y expresan un modo desadaptativo de resolver conflictos, con una orientación al sí mismo, al decir de Millon (1999). Se caracterizan por la inhibición, alteraciones en el pensamiento, en el desarrollo y en la autonomía, retraimiento, depresión y ansiedad. Mientras que los problemas externalizantes son comportamientos asociados a la expresión de conflictos emocionales con orientación hacia los demás (Millon, 1999), es decir, exteriorización de la agresión o descarga impulsiva (Achenbach, 2008).

El modelo de Achenbach utiliza datos provistos por una muestra significativa de niños; mediante técnicas de análisis factorial se determinaron agrupamientos de características de comportamiento. Este tratamiento estadístico asegura una elevada correlación entre los diversos comportamientos del niño. Lemos Giráldez (2003) señala que, teniendo en cuenta la co-ocurrencia de síntomas pertenecientes a diferentes entidades clínicas, es posible hipotetizar que dicha covariación obedece a la existencia de características comunes subyacentes a varios trastornos; por ende algunos diagnósticos representarían facetas de una misma dimensión de banda ancha, por ejemplo: en la adolescencia los trastornos de dependencia de sustancias, la conducta antisocial y el comportamiento desinhibido, compartirían mecanismos de vulnerabilidad propios de la dimensión externalizante. La propuesta de Achenbach ofrece la posibilidad de combinar los sistemas categoriales (banda angosta), con los sistemas dimensionales (banda ancha). Silva, Martínez Arias \& Ortet (2000) señalan que estos dos potentes agrupamientos de trastornos infantojuveniles expresan a nivel psicopatológico los polos desadaptativos de dos ejes básicos identificados por ellos: conducta prosocial versus antisocial y sociabilidad versus insociabilidad. 
Achenbach, junto a Edelbrock (1981) diseñó un instrumento de evaluación muy utilizado en el campo de la clínica, el Child Behavior Checklist $(C B C L)$. Se trata de un sistema de evaluación multiaxial que toma en cuenta la percepción de los padres, de los profesores, la dimensión cognitiva, las condiciones físicas y la evaluación del Psicólogo clínico. Esta propuesta supera a los sistemas clasificatorios clásicos y su mérito es haber aportado conceptos para la comprensión de la psicopatología del desarrollo y su potencial vinculación con la personalidad del adulto; en tanto se le critica la falta de énfasis en el contexto, a los contenidos que incluye - que resultan muy globales - y a los datos que provee que resultan subjetivos.

La interrogante gira en torno a cuál es la incidencia de esos patrones incipientes de respuestas emocionales en la aparición de trastornos en la infancia y su vinculación con la progresiva organización de lo que se denominará personalidad. Otro interrogante es la estabilidad de los rasgos a lo largo del tiempo. Así, mientras Eysenck (2007) y Costa \& McCrae (1987) argumentan la estabilidad de aquellos a lo largo de la infancia y adultez, Del Barrio (2005) plantea la conveniencia de ser cautelosos, puesto que se ha encontrado una baja estabilidad en las puntuaciones - al administrar pruebas - a lo largo del tiempo. Se está en presencia de un tema sobre el cual falta proseguir las investigaciones, ya que una línea de hipótesis apunta al cambio por acción del ambiente, y otra, a las limitaciones intrínsecas de la evaluación del constructo, puesto que son los padres o cuidadores los informantes.

\section{Una perspectiva integradora}

Millon afirma que ni la perspectiva nomotética - como la de Eysenck o la de Costa \& McCrae o Achenbach - ni la ideográfica - como la psicoanalítica - son satisfactorias. La limitación del enfoque nomotético es que no considera la singularidad del sujeto y el ideográfico no admite que cada sujeto puede ser comparado con otros. Aunque cada individuo tiene una historia única, dicha biografía, por si sola impide hacer generalizaciones, que el método científico exige. Con acierto Millon afirma que "...Los constructos no pueden existir sin individuos con los que ser comparados ni los individuos pueden ser entendidos sino a través de los constructos “(1999, pág.8).
Millon \& Davis (1999) proponen una integración de los enfoques nomotético e ideográfico y consideran que el concepto de sistema es óptimo para establecer un nexo entre ambas perspectivas. La personalidad se caracterizaría por su holismo, siendo necesario diferenciar diversos comportamientos de algunos principios organizadores que subyacen a aquellos. Esos principios nomológicos serían aplicables a todo organismo vivo como sistema biopsicosocial y se sustentan en actividades adaptativas, según Millon y Davis pueden sintetizarse en: existencia (placer-dolor); adaptación (activo-pasivo); relación (yo-otros) y abstracción (pensamiento-sentimiento).La teoría de Millon tiene el mérito de haber hecho una síntesis integradora entre diversos enfoques, como el psicoanalítico, el evolucionista, el biologicista o el factorialista, a los que considera muy doctrinales.

Es de destacar que Millon construye pruebas para evaluar la personalidad, pero a partir de los 13 años, como el Inventario Clínico de Personalidad para adolescentes (MACI o el Inventario de Personalidad de Millon para adolescentes (MAPI); para adultos el Inventario Clínico Multiaxial (MCMI -III) y el Inventario de Estilos de Personalidad (MIPS). De ello se infiere que, hasta la adolescencia no sería posible, o no sería prudente conceptualizar la personalidad, paso previo a la construcción de pruebas de evaluación de esta. En cambio, hace contribuciones muy valiosas acerca de cómo el temperamento, desde el momento del nacimiento, sería un modo preferente de respuestas, que operarían como base de la configuración de lo que posteriormente se denominará personalidad.

\section{Clasificación de los trastornos de la infancia y adolescencia.}

¿Son útiles?. Ventajas y riesgos

Puede afirmarse que los sistemas clasificatorios, tales como los publicados por la Organización Mundial de la Salud (OMS, 1999), denominados CIE, los editados por la American Psychiatric Association -DSM (2002)- o la Clasificación China de Desórdenes Mentales de la Chinese Society of Psychiatry (CCMD-3, 2001) - en sus sucesivas revisiones y basados en la tradición kraepeliana responden a una posición universalista.

Plantean que es posible construir categorías aplicables en diversos contextos. Se trata de sistemas de diagnóstico estandarizado que ofrecen una clasificación categorial de los trastornos mentales 
basándose en criterios bien definidos. El propósito es lograr una mayor fiabilidad; así, el principal requisito para incluir un patrón de comportamiento en el DSM, por ejemplo, es que genere un síntoma perturbador (distress) o discapacidad (dissability) (Tanaka-Matsumi \& Draguns, 1997).

Si bien los sistemas clasificatorios se inscriben dentro de una concepción universalista, el CCMD-3 admite le incidencia de la cultura en determinados trastornos propios de la cultura china. En el caso del DSM- IV se incluyó una Apéndice $(\mathrm{J})$ con lo que se denominó síndromes culturalmente limitados (Contini, 2013). En la $5^{\circ}$ edición del DSM (2014), dichos síndromes pasaron a denominarse conceptos culturales del malestar. Igualmente se agregó una Entrevista para tener en cuenta las variables culturales. Para quienes adscriben a un enfoque ideográfico de la personalidad, desde una perspectiva extrema, las taxonomías de la personalidad, tales como el DSM presentan profundas limitaciones en cuanto a su capacidad explicativa.

Este modelo ha sido resistido desde la comunidad de psicólogos. Más aún si se los analiza desde una mirada de clínica psicoanalítica. Esas resistencias se han visto agudizadas más en niños que en adultos, debido a la condición estática de las referidas categorías para ser aplicadas a un niño /adolescente en desarrollo. Las críticas se centran en las consecuencias del etiquetado y la falta de sensibilidad al contexto (Jiménez Hernández, 1995; Contini, 2013; Tanaka-Matsumi \& Draguns, 1997).

No obstante ello, han persistido a lo largo del tiempo e inclusive son de uso obligatorio para que la prestación que realiza el psicólogo (diagnóstico) sea reconocida por los seguros de salud. A favor de los sistemas clasificatorios se puede argumentar que han creado un lenguaje común que permite la comunicación entre los diversos integrantes de equipos de salud mental. (Contini, 2013).

En contraposición a este modelo de clasificación en cientos de categorías, se fue desarrollando otro, con base empírica y clínica, aplicable a la infancia, al cual se hizo referencia en apartados anteriores. Se trata del Modelo de Achenbach (1990).

Ahora bien, se consideran particularmente adecuados los interrogantes planteados por Jiménez Hernández (1995), referidos al valor de focalizar en la identificación de conductas simples y aisladas, o si es más relevante diferenciar patrones de comportamiento. Se está haciendo referencia aquí a agrupamientos de problemas. El segundo interrogante es si ambas son posturas excluyentes $\mathrm{o}$, por el contrario, complementarias.

\section{A modo de Síntesis}

Uno de los objetivos de esta comunicación ha sido conceptualizar la psicopatología infantojuvenil y dilucidar algunas cuestiones que se tornan sustanciales en el proceso de evaluación psicológica; lo que será imprescindible para identificar el grado de ajuste del comportamiento del niño/adolescente según lo que se espera para su edad cronológica y contexto ecológico cultural. A la Psicopatología se la conceptualizó como una disciplina que se propone estudiar las organizaciones y dinámicas psicológicas que subyacen a las perturbaciones del psiquismo, con el propósito de conocer sus determinantes, evolución y consecuencias que un trastorno puede generar en el desarrollo del niño/adolescente.

Se hizo referencia a la importancia de la combinación de estudios transversales con estudios longitudinales y se desarrollaron conceptos centrales para poder esclarecer cuándo se está en presencia de un comportamiento normal o patológico. Se destacó que se han de tener en cuenta tres aspectos primordiales: frecuencia, duración e intensidad del comportamiento, lo que conduce a analizar si ocurren por exceso o por defecto. Se planteó así también el análisis del comportamiento en el contexto del desarrollo, la cronodependencia, la valoración de este según el nivel cognitivo alcanzado por el niño/adolescente, el sesgo de los multinformantes, en particular de padres y maestros; el análisis del comportamiento según la situación y el progresivo peso que se atribuye a los factores culturales. En tal sentido la interrogante gira en torno al papel de la familia, la escuela, el grupo de pares y la comunidad misma en la generación de salud o psicopatología en la infancia y adolescencia.

Un segundo objetivo fue analizar cómo situar los incipientes trastornos psicopatológicos en infancia y adolescencia y así se definieron los conceptos de temperamento, carácter y personalidad. Se analizó si era posible emplear la noción de personalidad en la Infancia. Para ello se sometieron a análisis algunas teorías como las de Eysenck y McCrae y Costa de las que se han derivado pruebas para evaluar personalidad en niños. 
Se tuvieron también en cuenta los estudios de Achenbach quien, si bien no ha estado interesado en el tema de la personalidad, hace aportes sustanciales a la comprensión de las primeras organizaciones emocionales de niño (internalizante- externalizante). Tales consideraciones son muy valiosas para ahondar en la incidencia de esos patrones incipientes de respuestas emocionales en la configuración de trastornos psicopatológicos posteriores.

Por último, se tuvieron en cuenta las conceptualizaciones de Millon, por su carácter integrador entre los enfoques- a nuestro criterio parciales denominados nomotéticos e ideográficos.

Así también se discutió la conveniencia, alcances y riesgos de utilizar categorías diagnósticas estandarizadas para designar trastornos psicopatológicos en la infancia y adolescencia.

Las líneas de investigación que se desprenden de este trabajo se orientan mayoritariamente a realizar estudios longitudinales, que permitan superar las posiciones que plantean la estabilidad del temperamento a lo largo del tiempo y las que sostienen la variabilidad de este. Otra línea de investigación se vincula a la psicopatología del desarrollo, planteándose la necesidad de confirmar las relaciones de continuidad-discontinuidad a largo plazo entre las organizaciones psicopatológicas del niño y el adulto.

Se buscó perfilar una respuesta provisional -a una pregunta central- respecto a si es posible conceptualizar la personalidad en la infancia. Si se la considera como una estructura, una matriz producto de la relación temperamento /ambiente - se concluye que sería factible a partir de la adolescencia, cuando ya no tuvieran tanta incidencia las variables del desarrollo. Eso explicaría por qué las pruebas para niños disponibles responden a teorías dimensionales

Finalmente se puede afirmar que tanto la psicopatología como los estudios sobre personalidad en la infancia son nuevos, y que, si bien muestran importantes avances, en su gran mayoría se encuentran aún en estado de desarrollo. Se trata, en suma, de dos pilares para que las tareas de evaluación psicológica sean más rigurosas y estén respaldadas en teorías potentes y en evidencias. En la infancia y adolescencia la evaluación y diagnóstico psicológico adquiere un valor relevante, ya que brinda conceptos imprescindibles para generar acciones de prevención cuyo fin último es el logro de una posterior vida saludable.

\section{Referencias}

Achenbach, T \& Edelbrock, C. (1981). Behavioral problems and competences reported by parents of normal and disturbed aged 4 through 16. Monographs of the Society for Research in Child Development, 46, 1-82.

Achenbach, T. (1992). New developments in multiaxial empirically based assessment of child and adolescent psychopathology. En J. Rosen \& P. McReynolds (Dir.), Advances in Psychological Assessment, 8 (pp. 75-102). New York: Plenum.

Achenbach, T. (2008). Assessment, diagnosis, nosology and taxonomy of child and adolescent psychopathology. In Hersen \& Gross (Eds.) Handbook of clinical psychology,(pp.15-48). New York, USA: John Wiley \& Sons Inc.

Almonte, C. (2012). Características generales de la psicopatología infantil y de la adolescencia, en Almonte, C. y Montt, M. E., Psicopatología infantil y de la adolescencia, (pp. 114-125). Santiago de Chile: Mediterráneo.

American Psychiatric Association (APA) (2002). Manual diagnóstico y Estadístico de los trastornos mentales, DSM IV-TR. Criterios diagnósticos. Breviario. Barcelona: Masson.

Asociación Americana de Psiquiatría (APA). (2014). Guía de Consulta de los criterios diagnósticos del DSM 5. Madrid: Editorial Médica Panamericana.

Bates, J. \& Bayle, K. (1988). The role of the attachment in the development of behavior problems. En J. Belsky y T. Nezworski (Eds), Clinical Implications of Attachment (pp. 253-299). New York: Erlbaum.

Berry. J., Poortinga, Y, Breugelmans, S., Chasiotis, A. \& Sam, D. (2011). Cross-Cultural Psychology. Research and Applications, (3rd edition). UK, Cambridge: Cambridge University Press.

Campbell, S (1990). The socialization and social development of hiperactive children. En M. Lewis y S. Miller (Eds), Handbook of developmental psychopatology (pp.77-91). New York: Plenum Press.

Castro Solano, A. (2015). La aproximación teórico-racional: el modelo de T. Millon en M. Fernández Liporace y A. Castro Solano (Comp.), Evaluación de la personalidad normal y sus trastornos, (pp.15-47), Buenos Aires: Lugar.

Chinese Society of Psychiatry (2001). The third edition of The Chinese Classification and Diagnostic Criteria of Mental Disorders (in Chinese. CCMD-3) Jinan: Shandong Science and Technology Press.

Contini, N. (2013). La Evaluación Psicológica en debate. Aportes desde la Psicología Transcultural, Conferencia por invitación. Libro de Actas. XXXIV Congreso Interamericano de Psicología, Brasilia, 15-19 julio. Sociedad Interamericana de Psicología (SIP): 165-178.

Del Barrio Gándara, V. (2005). Temperamento, en L. Ezpeleta (Ed). Factores de riesgo en psicopatología del desarrollo (pp 113-145). Barcelona: Masson.

Eysenck, H \& Eysenck, S. (2007). EPQ. Cuestionario de personalidad para niños. (EPQ-J) y adultos (EPQ-A). (12 ${ }^{\mathrm{a}}$ Edición). Madrid: TEA.

Eysenck, H. (1992). Four ways five factors re not basic, Person. \& Indiv. Diffs, 13(6), 667-673.

Georgas, J, van de Vijver, F. \& Berry, J. (2004). The ecocultural framework, ecosocial indices and psychological variables in cross-cultural research. Journal of Cross Cultural Psychology, 35, 71-96.

Goldsmith, H. \& Gottesman, I. (1981). Origins of variation in behavioral style: A longitudinal study of temperament in young twins .Child Development, 52, 91-103. 
González Barrón, R. \& Montoya-Castilla, I. (2015). Psicología clínica infanto-juvenil. Madrid: Pirámide.

Jiménez Hernández, M. (1995). Psicopatología Infantil. Málaga: Aljibe.

Kagan, J. (1989.) Temperamental contributioin to social behavior. American Psychologist, 44, 668-674.

Lemos Giraldez, S. (2003). La psicopatología de la infancia y la adolescencia: consideraciones básicas para su estudio. Papeles del Psicólogo, 24(85), 19-28.

Lemos, V. (2006). La evaluación de la personalidad infantil a partir de enfoque de los Cinco Grandes Factores de personalidad (Big Five). Psicodiagnosticar, 16, 97-108.

Luciano, M. (Ed). (1997). Manual de Psicología clínica. Infancia y adolescencia. Valencia: Promolibro.

Mathiesen, K. \& Tabs K. (1999). The EAS Temperament questionnaire factor structure, age trends, reability and stability in a Norwegian sample. Journal of Child Psychology and Psychiatry, 40, 431-439.

McCrae, R. \& Costa, P. (1987).Validation of the Five-Factor Model of personality across instruments and observers. Journal of Personality and Social Psychology, $52,81-90$

Millon, T, Krueger R. F. \& Simonsen, E. (2010). Contemporary directions in psychopathology. New York: The Guilford Press.

Millon, T. \& Davis, R. (1999). Trastornos de la personalidad más allá del DSM IV. Masson: Barcelona.

Millon, T. \& Grossman, S. (2001). Overcoming Resistant Personality Disorders: A Personalized Psychotherapy Approach. New York: John Wiley \& Sons.

Millon, T. (1990).Toward a new personology: An evolutionary model. New York: John Wiley \& Sons.

Organización Mundial de la Salud (OMS) (1999). Trastornos mentales y del comportamiento de la décima revisión de la Clasificación Internacional de las Enfermedades (CIE-10), Ginebra.

Páez, D. y Casullo, M. (2000). Cultura y alexitimia. Buenos. Aires: Paidós.
Rodríguez Sacristán, J, Lozano Oyola, J. \& Rodríguez- Sacristán Cascajo, A. (2005). La constitución conceptual de la psicopatología infantil. Las claves del hecho clínico psicopatológico infantil, en Rodríguez Sacristán, J. (Dir.) (2005) Psicopatología infantil básica (pp. 45-48). Madrid: Pirámide.

Ross, A. (1980). Psychological disorders of children: A behavioral approach to theory, research and therapy (2nd edition). New York: McGraw. Hill.

Rothbart, M. \& Bates, J. (2006). Temperament. In W. Damon. R. Lerner \& N. Eisenberg (Eds). Handbook of child psychology, Vol 3. Social, emotional and personality development (pp. 99-166). New York: Wiley.

Rothbart, M. (2007). Temperament, Development, and Personality. Current Directions in Psychological Science. Association for Psychological Science, Vol 16(4).

Schoeps, K. \& González Barrón, R. (2015). Aproximación histórica a la psicología clínica infanto-juvenil, en R. González Barrón, R. y I. Montoya Castilla. Psicología Clínica Infanto-juvenil (pp 21-40). Madrid: Pirámide.

Silva, F., Martínez Arias., R. \& Ortet, G. (2000). Evaluación de la orientación interpersonal. En A. Cordero (Coord), La evaluación psicológica en el año 2000 (pp. 173-202). Madrid: TEA.

Sroufe, L. \& Rutter, M. (1984). The domain of developmental psychopathology. Child Development: 55, pp 17-29.

Tanaka-Matsumi, J. \& Draguns, J. (1997).Culture and Psychopathology. In W. Berry, M. Segall and C. Kagitçibasi (Eds), Handbook of Cross-Cultural Psychology, Vol III, Social Behavior and Applications (pp.449-491). Boston: Ma: Allyn / Bacon.

Thomas, A. Chess, S, \& Birch, G. (1968). Temperament and behavior disorder in children. New York: New York University Press. 